九州大学学術情報リポジトリ

Kyushu University Institutional Repository

\title{
Nonlinear Attractive and Reachable Sets Under Optimal Control in Three-Body Problem
}

Bando, Ma i

Department of Aeronautics and Astronautics, Kyushu University : Associate Professor

Scheeres, Daniel J.

Aerospace Engineering Sciences, Fellow AIAA : A. Richard Seebass Endowed Chair Professor

http://hdl. handle. net/2324/4479054

出版情報: Journal of guidance, control, and dynamics. 41 (8), pp.1766-1775，2018-04-30. American Institute of Aeronautics and Astronautics

バージョン :

権利関係 : 


\title{
Nonlinear Attractive and Reachable Sets under Optimal Feedback Control in the Hill Three-Body Problem
}

\author{
Mai Bando* \\ Kyushu University, 744 Motooka, Nishu-ku, Fukuoka 819-0395, Japan \\ Daniel J. Scheeres ${ }^{\dagger}$ \\ University of Colorado at Boulder, Boulder, Colorado 80309
}

\section{Introduction}

The invariant manifold structures associated with hyperbolic equilibrium points or periodic orbits in the restricted three-body are the modern basis for low-energy design of ballistic trajectories. The mathematics of these manifolds and their description have been applied in many different ways to enable efficient approaches to designing cost-free transfers, as summarized in [1-7]. These approaches generally only consider small propulsive maneuvers to enable connections between manifolds that may not intersect, with the fundamental goal of driving these maneuvers to zero $[8,9]$.

In a previous paper [10], we investigated how the use of manifolds could be extended by the application of low-thrust maneuvers, motivated by the demonstrated ability of low-thrust to enable new interplanetary mission designs [11-13]. That paper took a purely local approach to the problem, and only considered optimal control using the quadratic cost function along the linearized manifolds in the vicinity of hyperbolic equilibrium points and periodic orbits in the Hill 3-body problem. The linearized optimal control problem can be reduced to Ricatti equations and was solved completely, enabling the construction of a feedback control law. The specific approach taken was to find the attractive set of the target equilibrium point or periodic orbit for a fixed time, thus defining ellipsoids in phase space of constant cost. The time was then a free parameter, and we explored the solutions for both infinite time and finite time. This study revealed that the asymptotic form of the attractive set to an equilibrium point or a fixed point under optimal control is completely defined by its left unstable eigenvectors and a term inversely proportional to its unstable

\footnotetext{
*Associate Professor, Department of Aeronautics and Astronautics, mbando@aero.kyushu-u.ac.jp, Member AIAA.

${ }^{\dagger}$ A. Richard Seebass Endowed Chair Professor, Aerospace Engineering Sciences, Fellow AIAA.
} 
eigenvalue under the linearized assumption [10].

In the current paper we continue our investigations into how optimal feedback control can be used to extend the invariant manifold structure of the three-body problem and extend the idea of the attractive set of an equilibrium point or a periodic orbit to a nonlinear system. Solutions to the optimal transfer problem are obtained by solving the Hamilton-Jacobi-Bellman (HJB) equation, a nonlinear partial differential equation that is difficult to solve in general. For the infinite-time problem, many approaches to obtain the smooth solutions to the HJB equation have been proposed in the literature. A Galerkin spectral method is proposed to solve the HJB equation in [14]. A successive approximation method is proposed to transform the HJB equation into a sequence of a linear partial differential equations in [15]. Lukes obtained a power series solution to the HJB equation by expanding the dynamics, cost and control input in power-series [16].

For the finite-time problem, solving the HJB equation becomes more difficult to solve due to the nonlinear terminal constraints. Guibout and Scheeres [17] and Park and Scheeres [18] proposed a novel method to solve the two-point boundary value problem using generating functions of a canonical transformation where higher order solutions are obtained by a power series expansion of generating functions. Vadali and Sharma [19] presented a series solution method for finite-time problem by expanding the value function as a polynomial series of the states and Lagrange multipliers.

We consider both the finite and infinite time problem, and introduce a power series solution to the HJB equation analogous to the method followed in [16]. Given the solution to the linearized problem, the solution to the higher order corrections can be reduced to quadratures carried out recursively, and we carry out this solution procedure up to 4 th order for a set of specific examples.

Our analysis also explores the relation between the attractive set to an hyperbolic solution, and the reachable set which can be achieved starting from a given hyperbolic solution under low thrust control. While these sets are not equivalent, they share many similar elements and are solved by the same set of partial differential equations. In addition, we show that when the attractive set to a hyperbolic solution contains a certain periodic orbit, that the reachable set of that periodic orbit also contains the hyperbolic solution. We note that this is an efficient and natural way to construct transfers between periodic orbits that utilizes the manifold structure associated with these terminal orbits. We show a specific example of this approach to connect a near-Lyapunov orbit with a Halo orbit in the Hill 3-body problem.

The paper is structured as follows. We first review the attractive set of optimal control for linear system in Sec. 2. The reachability problem is also formulated and reachable set of optimal control is introduced. Section 3 provides the main theoretical results. In Sec. 4, examples are given to demonstrate the main results for an equilibrium point, a Lyapunov orbit and a halo orbit in the Hill three-body problem. Section 5 gives closing remarks. 


\section{Attractive sets of Optimal Control for Nonlinear System}

In this Section, the minimum energy problem with fixed terminal state for an affine nonlinear system is reviewed. Then the attractive and reachable set are introduced as the level set of the optimal cost.

\section{Problem statement}

Let us consider the minimum energy problem for an affine nonlinear system

$$
\begin{aligned}
& \dot{\boldsymbol{x}}=f(\boldsymbol{x}, t)+B \boldsymbol{u}, t \in \boldsymbol{T}=\left[0, t_{f}\right] \\
& J=\frac{1}{2} \int_{t_{0}}^{t_{f}}\|u\|^{2} d t
\end{aligned}
$$

subject to

$$
\boldsymbol{x}\left(t_{0}\right)=\boldsymbol{x}_{0}, \boldsymbol{x}\left(t_{f}\right)=\boldsymbol{x}_{f}
$$

where $\boldsymbol{x} \in \mathbb{R}^{n}$ is the state, $\boldsymbol{u} \in \boldsymbol{U} \subset \mathbb{R}^{m}$ is the control where $\boldsymbol{U} \subset \mathbb{R}^{m}$ is a set of admissible controls, $f(\boldsymbol{x}, t) \in \mathbb{R}^{n} \times \boldsymbol{T}$ is assumed to be continuous with respect to $t, B \in \mathbb{R}^{n} \times \mathbb{R}^{m}$ and $J$ is a performance index to be minimized. Moreover, we assume that the linearized system $\dot{\boldsymbol{x}}=A \boldsymbol{x}+B \boldsymbol{u}$ is controllable where $A$ is a first order approximation about a nominal solution of the non-linear differential equations, and that the state remains in the vicinity of this solution $[16,20]$.

\subsection{Attractive and reachable sets of optimal control}

In the following the attractive set and reachable set are introduced by setting different target sets shown in Fig. 1.

\section{Attractive set of optimal control}

The boundary condition of the attractive problem is given as

$$
\boldsymbol{x}\left(t_{f}\right)=\boldsymbol{x}_{f} \in \boldsymbol{X}_{\text {target }}
$$

where $\boldsymbol{X}_{\text {target }} \subset \mathbb{R}^{n}$ denotes a set of terminal states referred to as the target set. Then the attractive set of a given target set $\boldsymbol{X}_{\text {target }}$ for the system (1) is defined as a set of initial states satisfying an inequality constraint on the cost function $J^{*} \leq c$ where $J^{*}$ denotes the infimum of $J$. 
Definition 2.1. The attractive set $\mathscr{E}_{A}\left(t_{0}, t_{f}, \boldsymbol{X}_{\text {target }}, c\right)$, of the system (1), is defined as the set of all initial states $\boldsymbol{x}\left(t_{0}\right)$ for which there exists an optimal control input $\boldsymbol{u}^{*}(t) \in \boldsymbol{U}, t \in\left[t_{0}, t_{f}\right]$ and a corresponding trajectory $\boldsymbol{x}(t)\left(t \in\left[t_{0}, t_{f}\right]\right)$ of the system (1), such that $\boldsymbol{x}\left(t_{f}\right) \in \boldsymbol{X}_{\text {target }}$ and $J^{*} \leq c$.

If an initial state $x_{0}$ lies inside the attractive set $\mathscr{E}_{A}\left(t_{0}, t_{f}, \boldsymbol{X}_{\text {target }}, c\right)$, then the cost of the transfer from $\boldsymbol{x}_{0}$ to $\boldsymbol{x}_{f}$ is less than $c$. Therefore the set $\mathscr{E}_{A}\left(t_{0}, t_{f}, \boldsymbol{X}_{\text {target }}, c\right)$ represents the attractive region associated with the optimal control of cost $J^{*} \leq c$.

We define the Hamiltonian as

$$
\mathcal{H}(\boldsymbol{x}, \boldsymbol{p}, t)=\min _{\boldsymbol{u} \in \boldsymbol{U}}\left\{\frac{1}{2} \boldsymbol{u}^{T} \boldsymbol{u}+\boldsymbol{p}^{T} f(\boldsymbol{x}, \boldsymbol{u}, t)\right\}
$$

where $\boldsymbol{p}$ is adjoint vector. From the necessary condition for optimality,

$$
\boldsymbol{u}=-B^{T} \boldsymbol{p}
$$

It is well known $[21,22]$ that for any $t \in \boldsymbol{T}$, the boundary of the attractive set can be computed as the zero level set of the solution $V(\boldsymbol{x}, t)$ of the Hamilton-Jacobi-Bellman (HJB) equation,

$$
\frac{\partial V}{\partial t}+H\left(\boldsymbol{x}, \frac{\partial V}{\partial \boldsymbol{x}}, t\right)=0, \quad V\left(\boldsymbol{x}, t_{f}\right)=0
$$

where the final condition $V\left(\boldsymbol{x}, t_{f}\right)=0$ where $\boldsymbol{x} \in \boldsymbol{X}_{\text {target }}$ is the boundary of the target set $\boldsymbol{X}_{\text {target }}$. For the system (1), the HJB equation becomes

$$
\frac{\partial V}{\partial t}+\frac{\partial V}{\partial \boldsymbol{x}} f(\boldsymbol{x}, t)-\frac{1}{2} \frac{\partial V}{\partial \boldsymbol{x}} B B^{T} \frac{\partial V^{T}}{\partial \boldsymbol{x}}=0, \quad V\left(\boldsymbol{x}, t_{f}\right)=0
$$

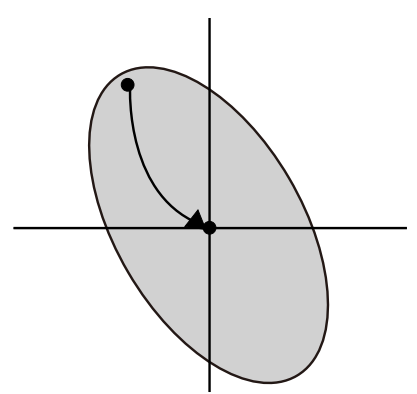

(a) Attractive set $\left(\boldsymbol{x}\left(t_{0}\right)=\boldsymbol{x}, \boldsymbol{x}\left(t_{f}\right)=0\right)$.

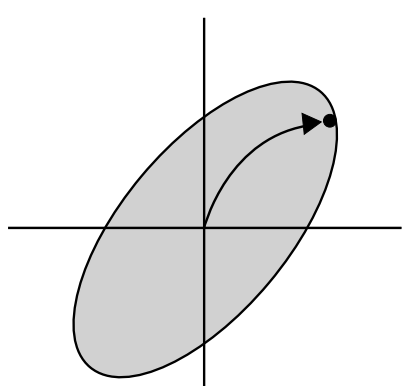

(b) Reachable set $\boldsymbol{x}\left(t_{0}\right)=0, \boldsymbol{x}\left(t_{f}\right)=\boldsymbol{x}$.

Fig. 1: Attractive set and reachable set. 


\section{Reachable set of optimal control}

The boundary condition for the reachable problem is given as

$$
\boldsymbol{x}\left(t_{0}\right)=\boldsymbol{x}_{0} \in \boldsymbol{X}_{\text {initial }}
$$

where $\boldsymbol{X}_{\text {initial }} \subset \mathbb{R}^{n}$ denotes a set of initial states referred to as the initial set. Then the reachable set (of a given initial set $\boldsymbol{X}_{\text {initial }}$ ) for the system (1) is defined as follows:

Definition 2.2. The reachable set $\mathscr{E}_{R}\left(t_{0}, t_{f}, \boldsymbol{X}_{\text {initial }}, c\right)$, of the system (1), is defined as the set of all terminal states $\boldsymbol{x}\left(t_{f}\right)$ for which there exists an optimal control input $\boldsymbol{u}(t), t \in\left[t_{0}, t_{f}\right]$ and a corresponding trajectory $\boldsymbol{x}(t)\left(t \in\left[t_{0}, t_{f}\right]\right)$ of the system (1), such that $\boldsymbol{x}\left(t_{0}\right) \in \boldsymbol{X}_{\text {initial }}$ and $J^{*} \leq c$.

If a terminal state $\boldsymbol{x}_{f}$ lies inside the reachable set $\mathscr{E}_{R}\left(t_{0}, t_{f}, \boldsymbol{X}_{\text {initial }}, c\right)$, then the cost of the transfer from $\boldsymbol{x}_{0}$ to $\boldsymbol{x}_{f}$ is less than $c$. Therefore the set $\mathscr{E}_{R}\left(t_{0}, t_{f}, \boldsymbol{X}_{\text {initial }}, c\right)$ defines the maximum reachable region associated with the optimal control of cost $J^{*} \leq c$.

By defining the Hamiltonian, the HJB equation for the reachable set is exactly as given in Eqs. (7) and (8) except the boundary condition becomes $V\left(\boldsymbol{x}, t_{0}\right)=0$ where $\boldsymbol{x} \in \boldsymbol{X}_{\text {initial }}$.

\section{Symmetry of the problems}

We note that the two problems are governed by the same equations, with the only significant difference being in the time direction the problem is solved in. From these symmetries in the attractive set and reachable set, if $\boldsymbol{x}_{0} \in \mathscr{E}_{A}\left(t_{0}, t_{f}, \boldsymbol{x}_{f}, c\right)$, then $\boldsymbol{x}_{f} \in \mathscr{E}_{R}\left(t_{0}, t_{f}, \boldsymbol{x}_{0}, c\right)$, and vice-versa. This implies that the same trajectory can solve both the attractive and reachable problem between two states. We use this symmetry later in constructing non-linear transfers between two periodic orbit solutions, which represents the most general application of our approach.

\section{Analytic expansion of Hamilton-Jacobi-Bellman equation}

In this section, we introduce the power series solution to the HJB equation, analogous to the method followed in [16].

\subsection{Calculation of Approximate Solutions for $u^{*}$ and $V$}

Assume that the target set $\boldsymbol{X}_{\text {target }}$ is represented by a nominal solution of (1) as $\boldsymbol{X}_{\text {target }}=\left\{\boldsymbol{\phi}_{0}(t) \in\right.$ $\left.R^{n} \mid \dot{\phi}_{0}=f\left(\phi_{0}, t\right)\right\}$. We define the relative state with respect to the nominal solution as $\boldsymbol{x}=\boldsymbol{\phi}-\boldsymbol{\phi}_{0}$. By 
expanding $f(\phi, t)$ about the nominal trajectory $\phi_{0}$ we find [18]

$$
\begin{aligned}
f(\phi, t) & =f\left(\phi_{0}, t\right)+\sum_{i}^{n} f_{i}\left(\phi_{0}, t\right) x_{i}+\sum_{i}^{n} \sum_{j}^{n} f_{i}\left(\phi_{0}, t\right) x_{i} x_{j}+\sum_{i}^{n} \sum_{j}^{n} \sum_{k}^{n} f_{i j k}\left(\phi_{0}, t\right) x_{i} x_{j} x_{k} \cdots \\
& =f\left(\phi_{0}, t\right)+f_{i}\left(\phi_{0}, t\right) x_{i}+f_{i j}\left(\phi_{0}, t\right) x_{i} x_{j}+f_{i j k}\left(\phi_{0}, t\right) x_{i} x_{j} x_{k}+\cdots
\end{aligned}
$$

where the summation convention is used in the second line and $f_{i}=\frac{\partial f}{\partial x_{i}}$. Therefore the relative motion can be described as

$$
\begin{aligned}
\dot{x} & =f(\boldsymbol{\phi}, t)-f\left(\boldsymbol{\phi}_{0}, t\right) \\
& =f_{i}\left(\phi_{0}, t\right) x_{i}+f_{i j}\left(\phi_{0}, t\right) x_{i} x_{j}+f_{i j k}\left(\phi_{0}, t\right) x_{i} x_{j} x_{k}+\cdots \\
& \equiv f^{(1)}(\boldsymbol{x}, t)+f^{(2)}(\boldsymbol{x}, t)+f^{(3)}(\boldsymbol{x}, t)+\cdots
\end{aligned}
$$

Since $f(\phi, t)-f\left(\phi_{0}, t\right)$ is a power series, $V$ is also expand as a power series in the relative state and is given by

$$
\begin{aligned}
V(\boldsymbol{x}, t) & =V_{i j}(t) x_{i} x_{j}+V_{i j k}(t) x_{i} x_{j} x_{k}+V_{i j k l}(t) x_{i} x_{j} x_{k} x_{l}+\cdots \\
& \equiv V^{(2)}(\boldsymbol{x}, t)+V^{(3)}(\boldsymbol{x}, t)+V^{(4)}(\boldsymbol{x}, t)+\cdots
\end{aligned}
$$

where it is well known that the leading term is quadratic. Then $u^{*}(\boldsymbol{x}, t)$ is given by

$$
\begin{aligned}
u^{*}(\boldsymbol{x}, t) & =-B^{T} \frac{\partial V^{(2)}}{\partial \boldsymbol{x}}(\boldsymbol{x}, t)-B^{T} \frac{\partial V^{(3)}}{\partial \boldsymbol{x}}(\boldsymbol{x}, t)-B^{T} \frac{\partial V^{(4)}}{\partial \boldsymbol{x}}(\boldsymbol{x}, t)-\cdots \\
& \equiv \boldsymbol{u}^{(1)}(\boldsymbol{x}, t)+\boldsymbol{u}^{(2)}(\boldsymbol{x}, t)+\boldsymbol{u}^{(3)}(\boldsymbol{x}, t)+\cdots
\end{aligned}
$$

Substituting Eq. (13) and (14) into the HJB equation (8) yields

$$
\begin{aligned}
\frac{\partial\left(V^{(2)}+V^{(3)}+\cdots\right)}{\partial t}+ & \frac{\partial\left(V^{(2)}+V^{(3)}+\cdots\right)}{\partial \boldsymbol{x}}\left(f^{(1)}(\boldsymbol{x}, t)+f^{(2)}(\boldsymbol{x}, t)+\cdots\right) \\
& -\frac{1}{2} \frac{\partial\left(V^{(2)}+V^{(3)}+\cdots\right)^{T}}{\partial \boldsymbol{x}} B B^{T} \frac{\partial\left(V^{(2)}+V^{(3)}+\cdots\right)}{\partial \boldsymbol{x}}=0
\end{aligned}
$$

The first order terms in Eq. (15) are given by

$$
\begin{aligned}
& \frac{\partial V^{(2)}}{\partial t}+\frac{\partial V^{(2)}}{\partial \boldsymbol{x}} f^{(1)}(\boldsymbol{x}, t)-\frac{1}{2} \frac{\partial V^{(2)}{ }^{T}}{\partial \boldsymbol{x}} B B^{T} \frac{\partial V^{(2)}}{\partial \boldsymbol{x}}=0, \quad V\left(\boldsymbol{x}, t_{f}\right)=0 \\
& \Leftrightarrow\left[\dot{V}_{i j}(t)+f_{i}\left(\boldsymbol{\phi}_{0}, t\right) V_{i j}(t)+V_{i j}(t) f_{j}\left(\boldsymbol{\phi}_{0}, t\right)-\frac{1}{2} V_{i j}(t) B B^{T} V_{i j}(t)\right] x_{i} x_{j}=0,
\end{aligned}
$$


and

$$
\boldsymbol{u}^{(1)}(\boldsymbol{x}, t)=-B^{T} \frac{\partial V^{(2)}}{\partial \boldsymbol{x}}(\boldsymbol{x}, t)=-B^{T} V_{i j} x_{j}
$$

Similarly, balancing terms of the same order in the power series expansion, the $m$-th order terms from Eq. (15) and $k$-th order terms from Eq. (14), the HJB equation is transformed into a system of ordinary differential equations for the coefficients.

$$
\begin{aligned}
\frac{\partial V^{(m)}}{\partial t}+\frac{\partial V^{(m)}}{\partial \boldsymbol{x}}\left(f^{(1)}-B \boldsymbol{u}^{(1)}\right)= & -\sum_{k=3}^{m-1} B \boldsymbol{u}^{(m-k+1)} \cdot \frac{\partial V^{(k)}}{\partial \boldsymbol{x}}-\sum_{k=2}^{m-1} f^{(m-k+1)} \cdot \frac{\partial V^{(k)}}{\partial \boldsymbol{x}} \\
& -\sum_{k=2}^{[(m-1) / 2]} \boldsymbol{u}^{(k)} \cdot \boldsymbol{u}^{(m-k)}-\frac{1}{2} \boldsymbol{u}^{(m / 2)} \cdot \boldsymbol{u}^{(m / 2)} \quad(m=3,4,5, \cdots) \\
\boldsymbol{u}^{(k)} & =-B^{T} \frac{\partial V^{(k+1)}}{\partial \boldsymbol{x}} \quad(k=2,3, \cdots)
\end{aligned}
$$

with boundary condition $V^{(m)}\left(\boldsymbol{x}_{f}, t_{f}\right)=0$. Starting with $\boldsymbol{u}^{(1)}$ and $V^{(2)}$, the higher order terms can be computed successively by solving Eqs. (19) and (20). Hence a power series solutions for $V$ and $\boldsymbol{u}$ can be obtained.

For the infinite-time problem, $\frac{\partial V^{(m)}}{\partial t} \rightarrow 0$ and Eq. (19) reduces to a set of linear algebraic equations

$$
\begin{aligned}
\frac{\partial V^{(m)}}{\partial \boldsymbol{x}}\left(f^{(1)}-B \boldsymbol{u}^{(1)}\right)= & -\sum_{k=3}^{m-1} B \boldsymbol{u}^{(m-k+1)} \cdot \frac{\partial V^{(k)}}{\partial \boldsymbol{x}}-\sum_{k=2}^{m-1} f^{(m-k+1)} \cdot \frac{\partial V^{(k)}}{\partial \boldsymbol{x}} \\
& -\sum_{k=2}^{[(m-1) / 2]} \boldsymbol{u}^{(k)} \cdot \boldsymbol{u}^{(m-k)}-\frac{1}{2} \boldsymbol{u}^{(m / 2)} \cdot \boldsymbol{u}^{(m / 2)} \quad(m=3,4,5, \cdots) \\
\boldsymbol{u}^{(k)} & =-B^{T} \frac{\partial V^{(k+1)}}{\partial \boldsymbol{x}} \quad(k=2,3, \cdots)
\end{aligned}
$$

The process of deriving equations for $V$ can be continued to arbitrarily high-order by the use of a symbolic manipulation program. In the following, the successive equations are solved by Mathematica (Wolfram Research, Inc.) In practical, the optimal control for the long time duration case is known to be sensitive to the numerical error. Therefore, the transient behavior of $\left.V^{(} m\right)(t)$ is ignored and the periodic feedback gain is used in the simulation. 


\subsection{Solutions for Linearized System}

The key to solving the recursive equations is to first have a linear solution, which we work out in detail in the following.

\section{Attractive set for a linear system}

For the second order solution, redefine $V$ and $f$ by the use of block matrices:

$$
\begin{array}{r}
V(\boldsymbol{x}, t)=V_{i j}(t) x_{i} x_{j} \triangleq \frac{1}{2} \boldsymbol{x}^{T} X(t) \boldsymbol{x} \\
f(\boldsymbol{x}, t)=f_{i}\left(\boldsymbol{\phi}_{0}, t\right) x_{i}=A(t) \boldsymbol{x}
\end{array}
$$

The second order solution of $V(\boldsymbol{x}, t)$ defines the ellipsoidal set as the attractive set of optimal control. The shape of the ellipsoid can be described by the eigenvalues and eigenvectors of the controllability grammian, which then extends to the non-linear system, In the following, we restate without proof the key result from [10] concerning how the linear structure around the target state organizes the attractive set.

Theorem 3.1 (Attractive set [10]). The asymptotic form of the attractive set to an equilibrium point or a fixed point under optimal control is completely defined by its left unstable eigenvectors and a term proportional to its unstable eigenvalue.

\section{Reachable set for a linear system}

As noted in Section 2, the HJB equation for the reachable set is identical to that for the attractive set except the boundary conditions.

Similar to the attractive set, the second order solution of $V(x, t)$ defines the ellipsoidal reachable ! ! set and the following holds for the reachable problem.

Theorem 3.2 (Reachable set). The asymptotic form of the reachable set to an equilibrium point or a fixed point under optimal control is completely defined by its left stable eigenvectors and a term proportional to its stable eigenvalue.

Proof. From the discussion above, the reachable set is equivalent to the attractive set of the adjoint system. Moreover, the adjoint system inherits the conjugate structure from the original system, meaning that the stable manifold becomes the unstable manifold and vice versa. Therefore, from Theorem 3.1, the asymptotic form of the reachable set to an equilibrium point or a fixed point under optimal control is completely defined by the unstable eigenvectors and a term proportional to unstable eigenvalue of its adjoint system $\dot{\boldsymbol{x}}=-A^{T} \boldsymbol{x}$ or $\boldsymbol{x}(k+1)=-A^{T} \boldsymbol{x}(k)$. 


\section{Applications}

The specific solution process for obtaining higher order solutions changes depending on whether the target solution is an equilibrium point or a periodic orbit. In this section we explore these different solution procedures, using the Hill 3-body problem as an example problem. We note that all of these procedures can also be applied to the Restricted 3-body problem, or indeed to any astrodynamical system [23], however the detailed expansions are more complicated and would mask the essential nature of our approach.

\subsection{Hill three-body problem}

The Hill three-body problem represents the dynamics of two particles of arbitrary relative mass with respect to each other while being perturbed by a massive third body. The restricted Hill three-body problem can be viewed as a simplification of the circular restricted three-body problem by assuming the first body has a larger mass than the second one and the third one has negligible mass [24]. It is in general a good model for approximating motion in the vicinity of the Earth for the spacecraft-Earth-Sun problem. The equations of motion in normalized form are given by

$$
\begin{gathered}
\ddot{x}-2 \dot{y}=\frac{\partial U}{\partial x}+u_{x} \\
\ddot{y}+2 \dot{x}=\frac{\partial U}{\partial y}+u_{y} \\
\ddot{z}=\frac{\partial U}{\partial z}+u_{z} \\
U=\frac{\mu}{r}+\frac{1}{2}\left(3 x^{2}-z^{2}\right), r=\sqrt{x^{2}+y^{2}+z^{2}}
\end{gathered}
$$

where the coordinate system is centered on the second body [23].

Introducing the state $\boldsymbol{x}=\left[\begin{array}{llllll}x & y & z & \dot{x} & \dot{y} & \dot{z}\end{array}\right]^{T}$ and control acceleration $\boldsymbol{u}=\left[\begin{array}{lll}u_{x} & u_{y} & u_{z}\end{array}\right]^{T}$, the equations of motion become

$$
\dot{\boldsymbol{x}}=f(\boldsymbol{x})+B \boldsymbol{u}, f(\boldsymbol{x})=\left[\begin{array}{c}
\dot{x} \\
\dot{y} \\
\dot{z} \\
2 \dot{y}+3 x-\frac{x}{r^{3}} \\
-2 \dot{x}-\frac{y}{r^{3}} \\
-\frac{z}{r^{3}}
\end{array}\right], B=\left[\begin{array}{ccc}
0 & 0 & 0 \\
0 & 0 & 0 \\
0 & 0 & 0 \\
1 & 0 & 0 \\
0 & 1 & 0 \\
0 & 0 & 1
\end{array}\right]
$$

Note that there are symmetries in the Hill problem $(x, y, z, \dot{x}, \dot{y}, \dot{z}, t) \leftrightarrow(x,-y, z,-\dot{x}, \dot{y},-\dot{z},-t)$. 


\subsection{Attractive and reachable sets for equilibrium point}

The Hill problem has two equilibrium solutions, the libration points located at $x= \pm\left(\frac{1}{3}\right)^{1 / 3}, y=z=0$. The eigenvalues of the linearized system are given by

$$
\begin{aligned}
& \lambda_{1,2}= \pm \sqrt{2 \sqrt{7}+1} \\
& \lambda_{3,4}= \pm j \sqrt{2 \sqrt{7}-1} \\
& \lambda_{5,6}= \pm 2 j
\end{aligned}
$$

The first four eigenvalues $\lambda_{1,2}$ and $\lambda_{3,4}$, correspond to the in-plane motion while the eigenvalues $\lambda_{5,6}$ correspond to out of plane motion. Note that the out-of-plane motion is decoupled in the linearized equation and consists only of periodic motions while the in-plane motion consists of a stable, an unstable and a center manifold.

For the planar motion of Eqs. (25)-(27), the attractive set of optimal control to transfer to the equilibrium point is considered, i.e. the target set is the equilibrium point

$$
\boldsymbol{X}_{\text {target }}=\left[\begin{array}{c}
\left(\frac{1}{3}\right)^{1 / 3} \\
0
\end{array}\right]
$$

The series expansion about $\boldsymbol{X}_{\text {target }}$ is given by

$$
\dot{\boldsymbol{x}}=f^{(1)}(\boldsymbol{x})+f^{(2)}(\boldsymbol{x})+\cdots+B \boldsymbol{u}
$$

where

$$
f^{(1)}(\boldsymbol{x})=\left(\begin{array}{c}
\dot{x} \\
\dot{y} \\
9 x+2 \dot{y} \\
-3 y-2 \dot{x}
\end{array}\right), f^{(2)}(\boldsymbol{x})=\left(\begin{array}{c}
0 \\
0 \\
\frac{9}{2} \sqrt[3]{3} y^{2}-9 \sqrt[3]{3} x^{2} \\
9 \sqrt[3]{3} x y
\end{array}\right), f^{(3)}(\boldsymbol{x})=\left(\begin{array}{c}
0 \\
0 \\
123^{2 / 3} x^{3}-183^{2 / 3} x y^{2} \\
\frac{9}{2} 3^{2 / 3} y^{3}-183^{2 / 3} x^{2} y
\end{array}\right), \cdots
$$

Note, $|x|,|y|,|\dot{x}|, \dot{y} \mid<<1$.

The nonlinear attractive and reachable sets to transfer to the origin was computed over a time interval $\left[0, t_{f}\right]$ in nondimensional time. First, the infinite-time problem is considered. In this case, the HJB equation is reduced to the algebraic equations for the unknown coefficients Eq. (21) and higher order solutions are successively obtained from $\boldsymbol{u}^{(1)}$ and $V^{(2)}$. Figure 2 shows the projection of attractive and reachable sets 


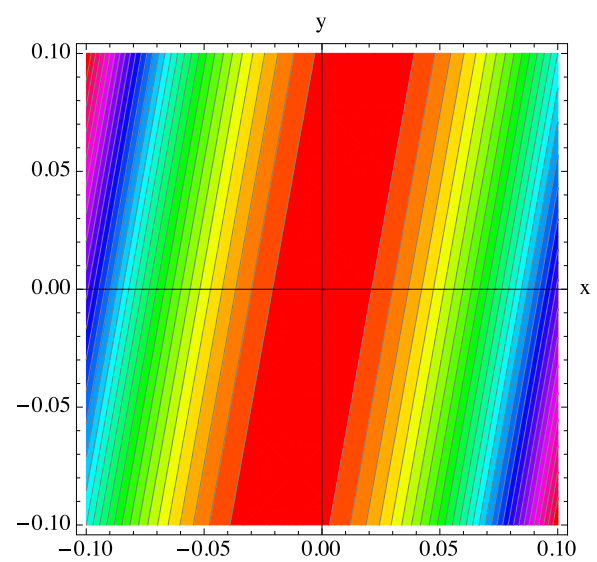

(a) Order 2.

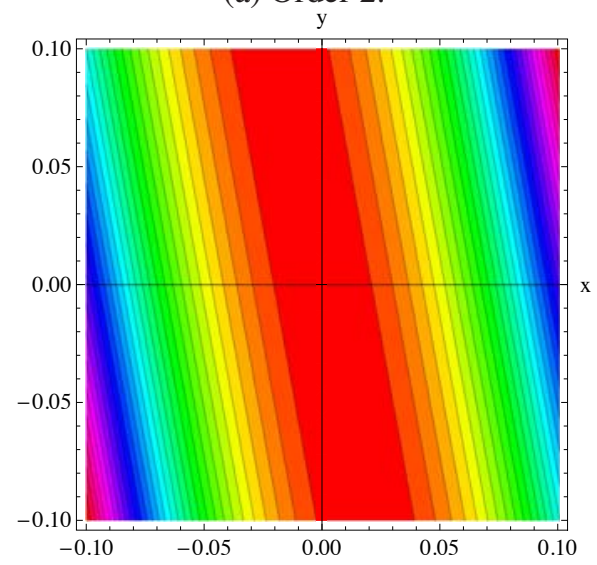

(d) Order 2.

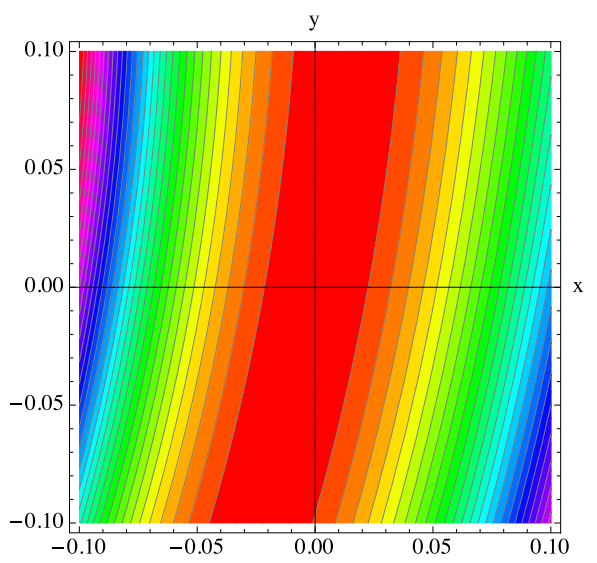

(b) Order 4 .

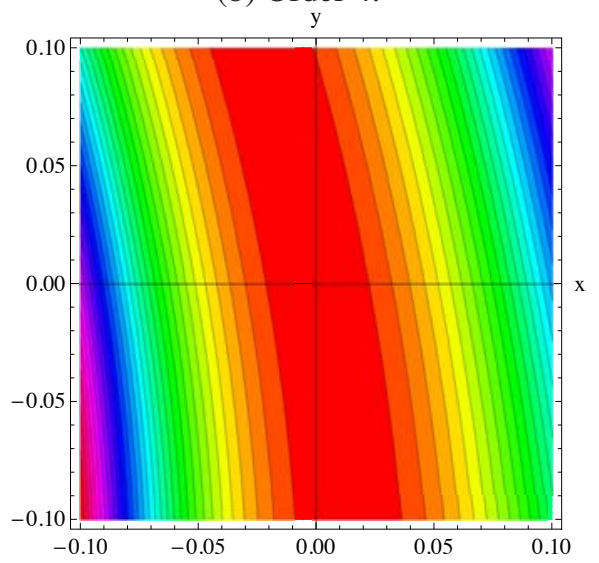

(e) Order 4 .

Fig. 2: Attractive set (top) and reachable set (bottom) of equilibrium point for $t_{f}=\infty$.

onto $x-y$ plane obtained by the power series solution of order 2 and 4 respectively. The solution to the linearized system is equivalent to the order 2 solution. The attractive and reachable sets for the infinite-time problem are unbounded along the right stable eigenvector and the least extent is along the left unstable eigenvector as stated in Theorem 3.1 and 3.2. This can be easily confirmed in the manifold coordinate (defined in [10]) in which the $x$-axis is aligned with the left unstable eigenvector and $y$-axis with the right stable eigenvector. The attractive set using the manifold coordinates $\left(x_{m}-y_{m}\right)$ is shown in Fig. 3 . It is noteworthy that the attractive set is unbounded along the right stable eigenvector $\left(y_{m}\right)$ for the higher order solution. Note that the attractive set and reachable set are symmetric with respect to the $y$-axis due to the symmetry properties of the Hill equations.

Next, the finite-time problem is considered. In this case, the HJB equation is reduced to the differential equations for the unknown coefficients and successively obtained by Eq. (19). Figures 4 - 5 show the the projections of attractive and reachable sets onto $x-y$ plane obtained by the power series solution of order 2 and 4 respectively. It can be seen that the higher order attractive set is almost the same as the ellipsoidal 


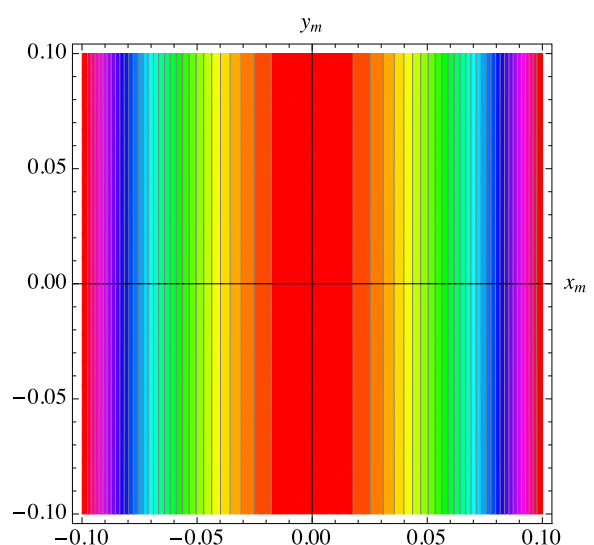

(a) Order 2.

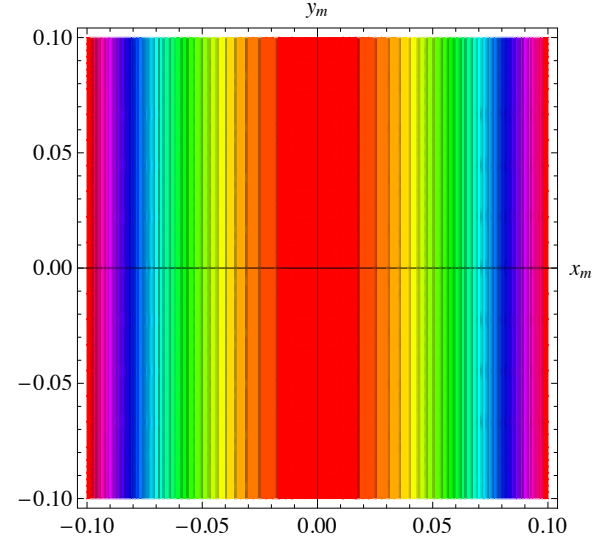

(c) Order 2.

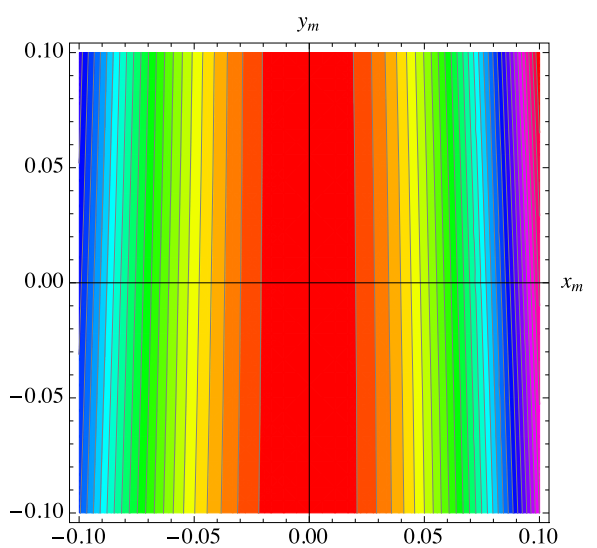

(b) Order 4.

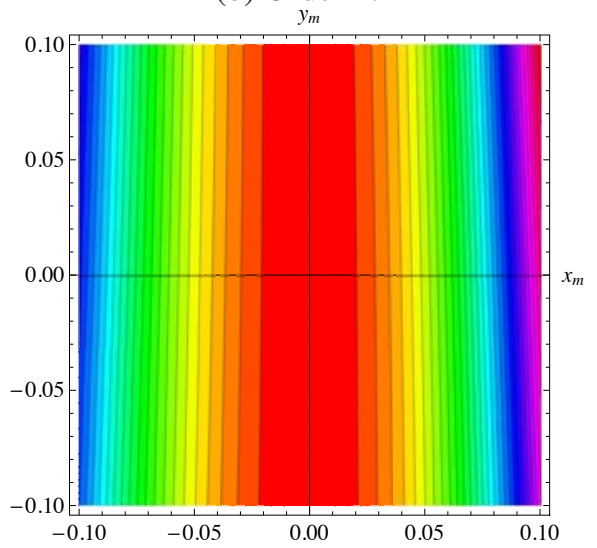

(d) Order 4 .

Fig. 3: Attractive set (top) and reachable set (bottom) of equilibrium point for $t_{f}=\infty$ in the manifold coordinate $\left(x_{m}-y_{m}\right)$.

solution of the second order problem for a short flight-time. For a longer flight-time, the shape of the contour of the attractive set is no longer ellipsoidal due to the nonlinearity.

\subsection{Attractive set of a fixed point: Lyapunov orbit case}

Next, the optimal control problem to transfer to a periodic orbit in the vicinity of the libration point is considered. Transfer problems can be solved in two ways: one uses the attractive set of the final Lyapunov orbit, and the other uses the reachable set of the initial Lyapunov orbit.

We assume that the spacecraft is initially on the Lyapunov orbit defined by the initial condition

$$
x_{\text {lyap } 0}(0)=\left[\begin{array}{llllll}
0.7640 & 0.0000 & 0.0000 & 0.0000 & -0.5211 & 0.0000
\end{array}\right]^{T}
$$




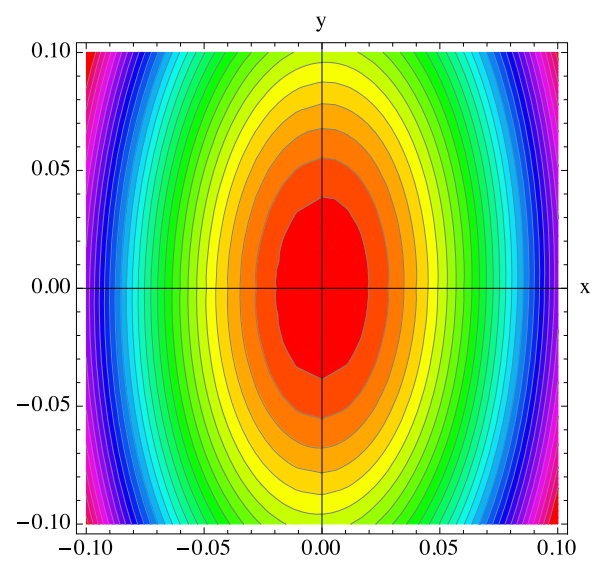

(a) Order 2.

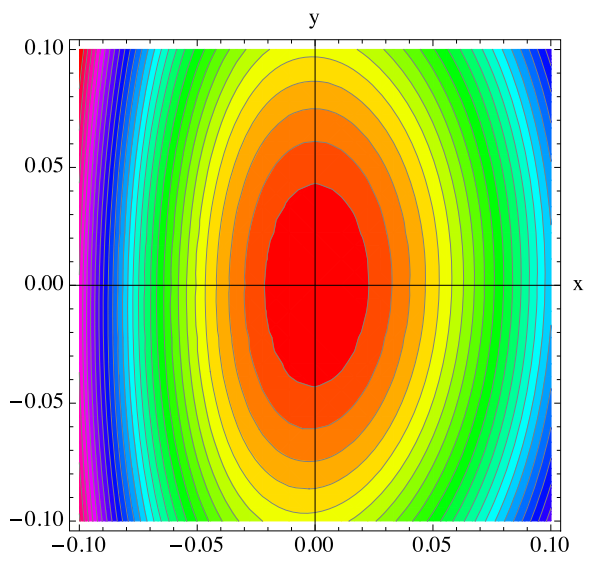

(b) Order 4.

Fig. 4: Attractive sets set of equilibrium point for $t_{f}=0.3$.

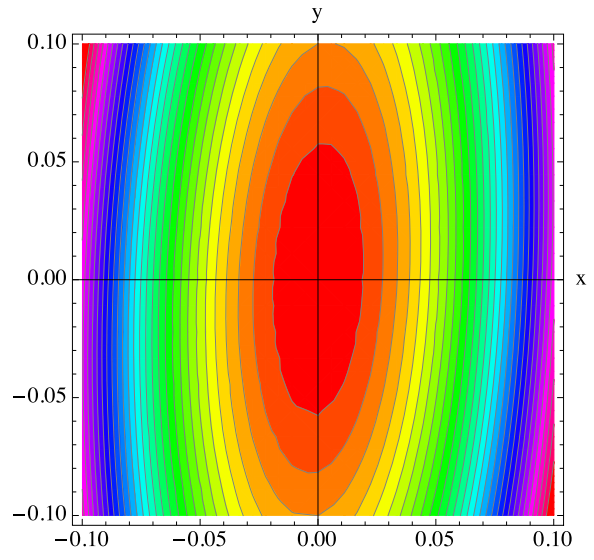

(a) Order 2.

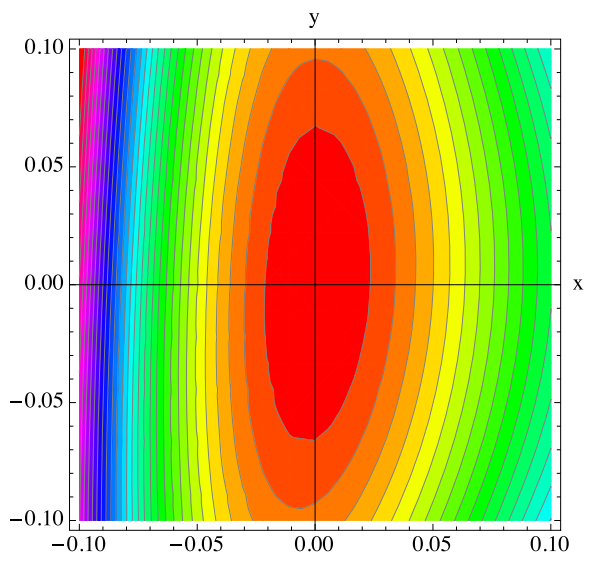

(b) Order 4.

Fig. 5: Attractive sets set of equilibrium point for $t_{f}=0.5$. 
and its period is $T_{0}=3.0669$ and the final Lyapunov orbit is defined as

$$
x_{\text {lyap }_{f}}(0)=\left[\begin{array}{llllll}
0.7600 & 0.0000 & 0.0000 & 0.0000 & -0.4895 & 0.0000
\end{array}\right]^{T}
$$

and its period is $T_{f}=3.0626$.

For the attractive problem, the target set (4) is defined by the final Lyapunov orbit as

$$
\boldsymbol{X}_{\text {target }}=\left\{\phi_{0}(t) \mid \dot{\phi}_{0}=f\left(\phi_{0}\right), \phi_{0}(0)=x_{\text {lyap }_{f}}(0)\right\}
$$

For the reachable problem, the target set (7) is defined by the initial Lyapunov orbit as

$$
\boldsymbol{X}_{\text {initial }}=\left\{\phi_{0}(t) \mid \dot{\phi}_{0}=f\left(\phi_{0}\right), \phi_{0}(0)=x_{\text {lyap }}(0)\right\}
$$

The nonlinear attractive and reachable sets to transfer to the origin was computed over a time interval $\left[0,100 T_{0}\right]$ in nondimensional time. The computation finds the coefficients for the attractive and reachable sets as continuous functions of time. For presentation purposes we only show them at stroboscopic periods of $t=k T, k=1,2, \cdots$. The series expansion about $\boldsymbol{X}_{\text {target }}$ is given by

$$
\dot{\boldsymbol{x}}=f^{(1)}(\boldsymbol{x}, t)+f^{(2)}(\boldsymbol{x}, t)+\cdots+B \boldsymbol{u}
$$

It should be noted that all coefficients of $f^{(1)}(\boldsymbol{x}, t), f^{(2)}(\boldsymbol{x}, t), f^{(3)}(\boldsymbol{x}, t), \cdots$ are $T$-periodic. Figure 6(a) shows the attractive set of the final Lyapunov orbit and Fig.6(b) shows the reachable sets of the initial Lyapunov orbit obtained by the power series solution of order 4 . The same optimal trajectory can be obtained by both attractive and reachable problem as shown in Fig. 7.

\subsection{Attractive set of the halo orbit}

The attractive set for a three-dimensional periodic orbit called a halo orbit is finally considered. Halo orbits are found by continuation from their bifurcation from planar Lyapunov orbits [23]. The particular halo orbit we used as our target is given by the normalized initial condition

$$
\boldsymbol{x}_{r e f}(0)=\left[\begin{array}{llllll}
0.7406 & 0.0000 & -0.3979 & 0.0000 & -0.8509 & 0.0000
\end{array}\right]^{T}
$$

with a period of $T=3.0461$. Figure 8 depicts this halo orbit in 3-dimensional position space, projected onto $x-y$ plane, $y-z$ plane and $x-z$ plane. This halo orbit has one pair of hyperbolic characteristic 


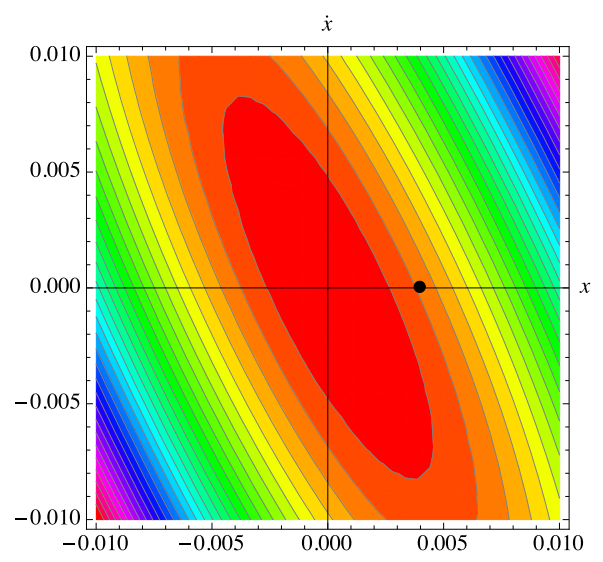

(a) Attractive set for Lyapunov orbit.

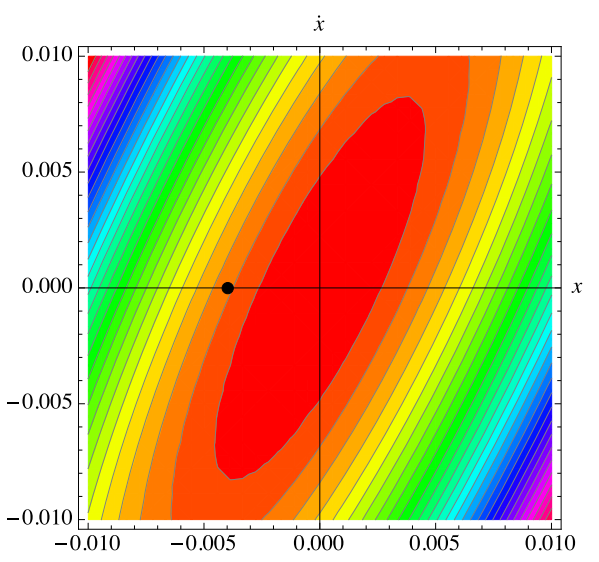

(b) Reachable set for Lyapunov orbit.

Fig. 6: Attractive set and reachable set of Lyapunov orbits: the origin represent the final (left) and initial (right) Lyapunov orbits and the black dot represent the initial (left) and final (right) Lyapunov orbits.

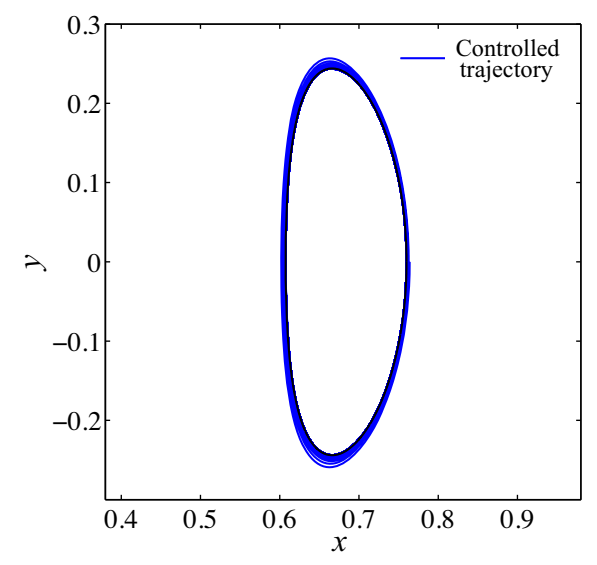

(a) Controlled trajectory in the $X-Y$ coordinate.

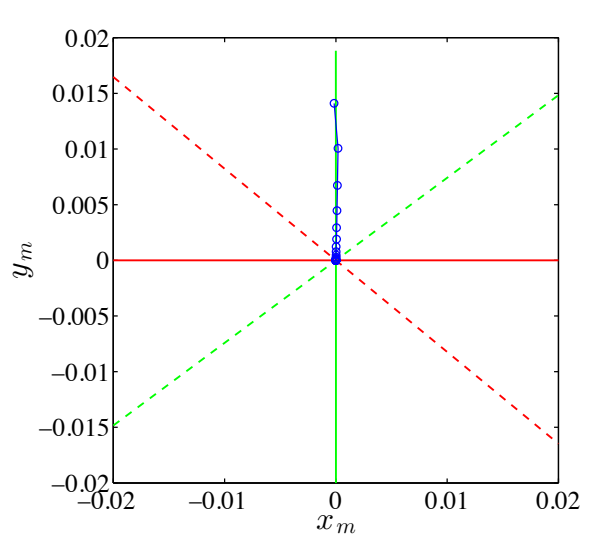

(b) Controlled trajectory in the manifold coordinate.

Fig. 7: Controlled trajectory.

exponents $\sigma= \pm 6.5918$ and a four dimensional center manifold.

As in the previous examples, the attractive set for the finite-time and infinite-time problem is obtained by solving the differential equations for the unknown coefficients successively obtained by Eq. (19). For the halo orbit case, the power series is obtained along the halo orbit and hence the HJB equation is reduced to a set of differential equations with time-periodic coefficients. Therefore, the solutions to the differential equation (19) converge in time to periodic solutions, in contrast to the equilibrium point case where they converge to a constant value. Figures 9 and 10 show the transient and steady-state periodic solution of the coefficient of $V^{(2)}$, and we note similar behavior for the higher order solutions. As a result, the attractive set for the periodic orbit (when viewed as a fixed point) converges to a similar structure. The attractive region becomes larger and finally becomes unbounded along the right stable eigenvector as in the previous examples. 


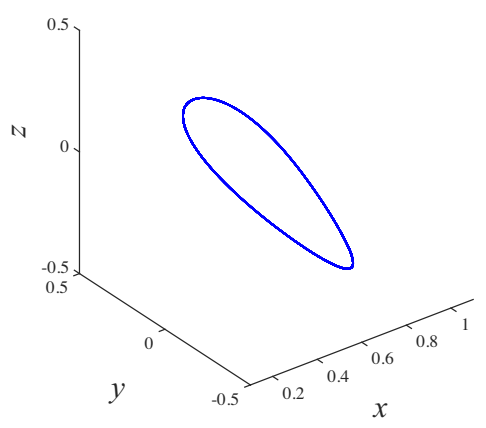

(a) 3D trajectory.

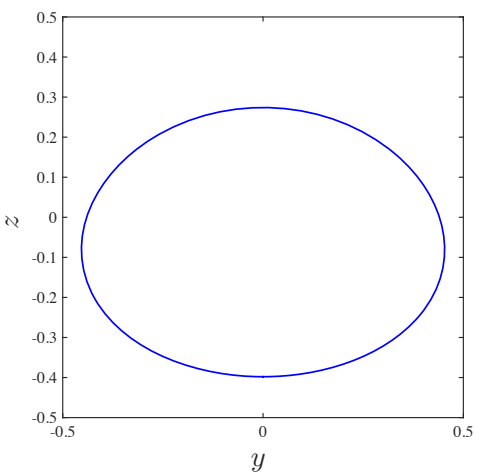

(c) Projection onto the $Y-Z$ plane.

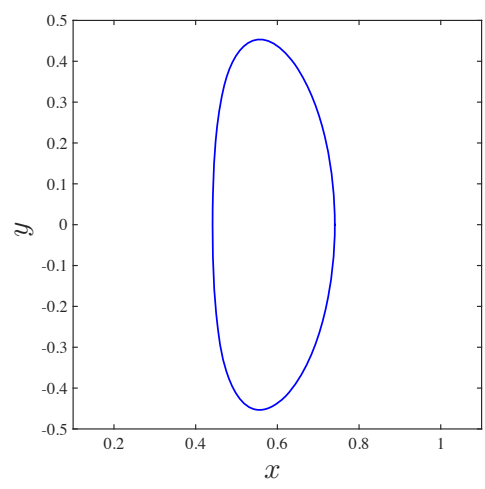

(b) Projection onto the $X-Y$ plane.

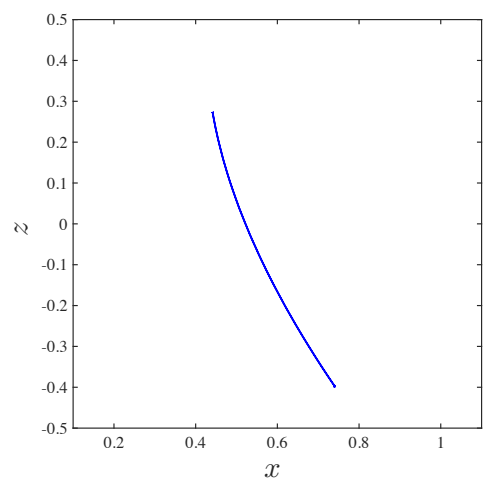

(d) Projection onto the $X-Z$ plane.

Fig. 8: Halo orbit.

As an application of the nonlinear attractive set, the optimal transfer between two different halo orbits is considered. We compute the nonlinear attractive set of a halo orbit and extend it to the point where we find a target halo orbit which lies inside a given level set. The point we choose for our example is defined by the initial condition

$$
\boldsymbol{x}(0)=\left[\begin{array}{llllll}
0.7747 & 0.0000 & 0.0001 & 0.0000 & -0.6138 & 0.0000
\end{array}\right]^{T}
$$

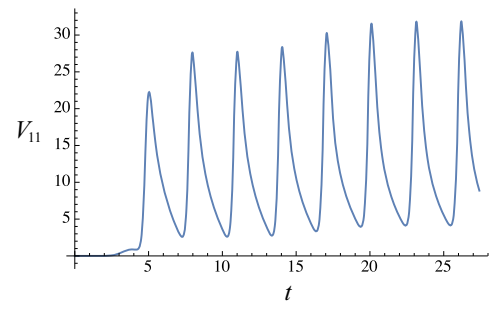

Fig. 9: Transient solution of $V_{11}(t)$ for halo orbit.

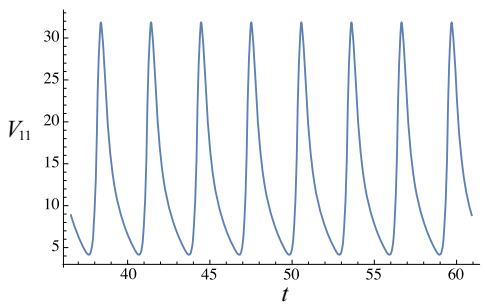

Fig. 10: Steady-state solution of $V_{11}(t)$ for halo orbit. 


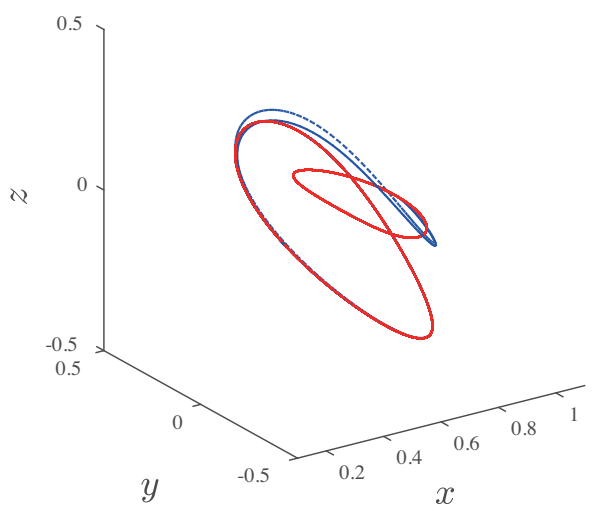

(a) 3D trajectory.

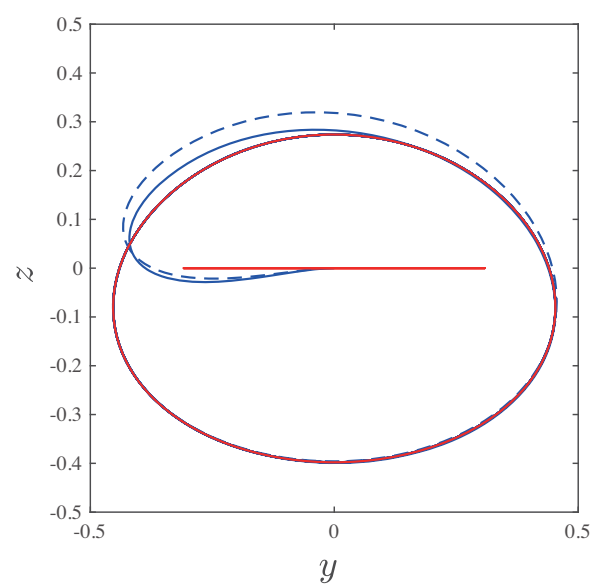

(c) Projection onto the $Y-Z$ plane.

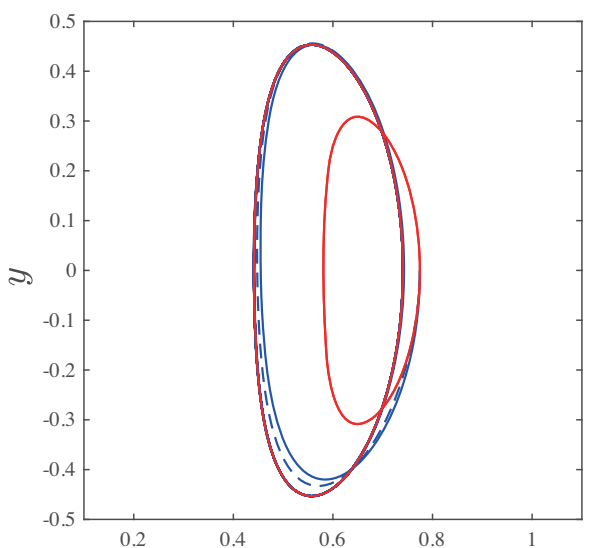

$x$

(b) Projection onto the $X-Y$ plane.

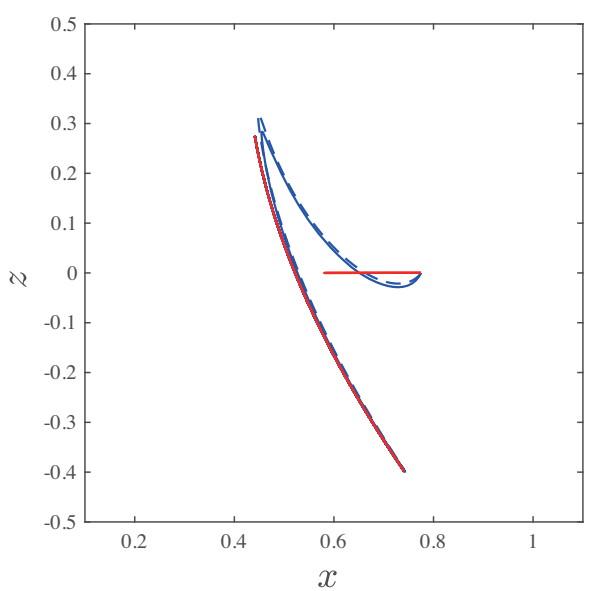

(d) Projection onto the $X-Z$ plane.

Fig. 11: Optimally controlled trajectory for $t_{f}=5 T$ (solid line: 4th order HJB, dotted line: feedback linearization).

and we note that for convenience we choose a very small out pf plane $z$-component. This point is inside the level set $c=0.12$ for the finite-time attractive set of $t_{f}=5 T$ and $c=0.06$ for $t_{f}=50 T$. The optimal control trajectories based on 4th order solution are shown in solid lines in Figs. 11 and 12 where $t_{f}=5 T$ and $t_{f}=50 T$ respectively.

For comparison, the nonlinear control approach using feedback linearization is implemented. The system can be written in the semi-linear form:

$$
\dot{\boldsymbol{x}}=A(t) \boldsymbol{x}+B(g(\boldsymbol{x})+\boldsymbol{u})
$$




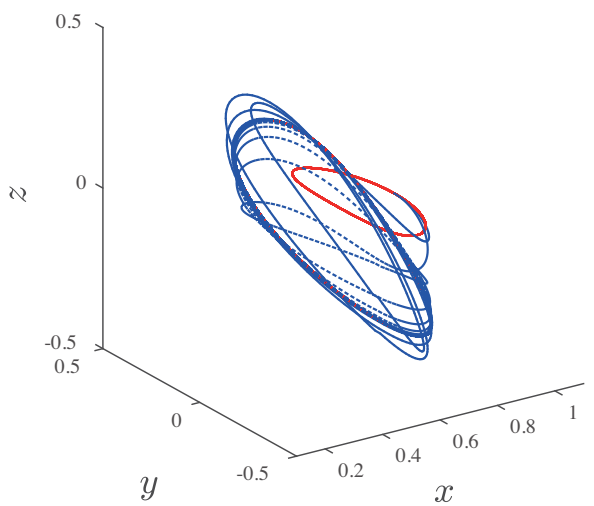

(a) $3 \mathrm{D}$ trajectory.

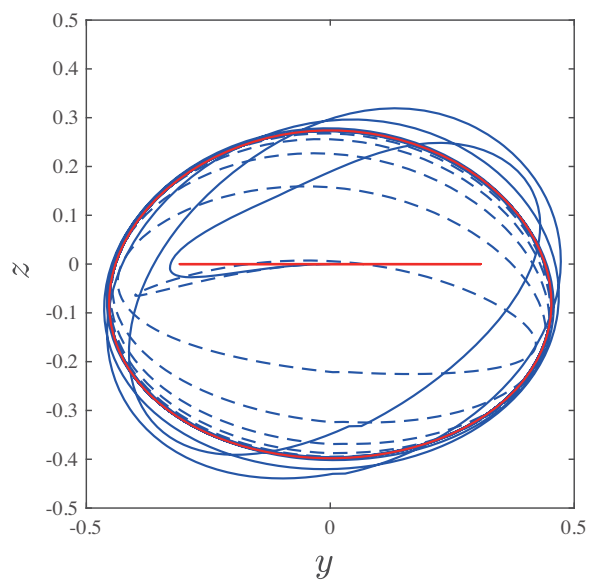

(c) Projection onto the $Y-Z$ plane.

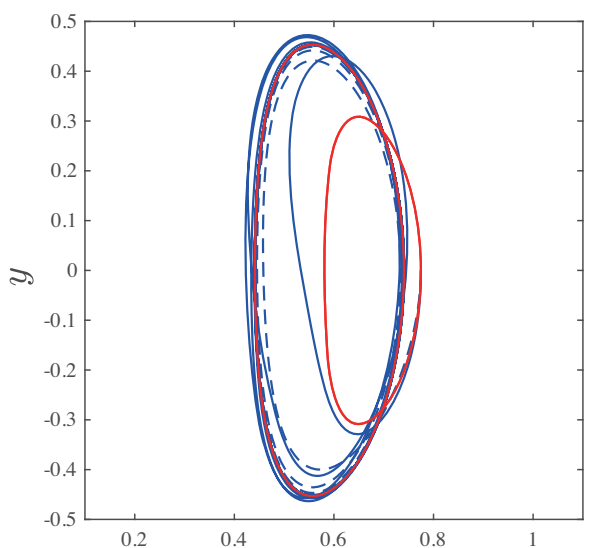

$x$

(b) Projection onto the $X-Y$ plane.

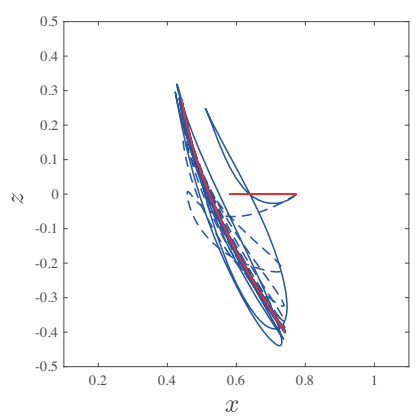

(d) Projection onto the $X-Z$ plane.

Fig. 12: Controlled trajectory for $t_{f}=50 T$ (solid line: 4th order HJB, dotted line: feedback linearization).

Then the nonlinear control input to decouple the system is given by

$$
\boldsymbol{u}=\boldsymbol{u}_{\text {linear }}-\boldsymbol{g}(\boldsymbol{x})
$$

The controlled trajectory based on Eq. (40) is shown in dotted lines in Figs. 11 and 12 where $t_{f}=5 T$ and $t_{f}=50 T$, respectively. To obtain the cost value, the integration along the controlled trajectory is required and those values were almost ten times larger than the optimal cost in the example. This example exemplifies the use of our proposed approach, as the cost function immediately defines the control and trajectory from any point in the attractive set to the target. 


\section{Conclusion}

This paper extends the idea of the attractive set of optimal feedback control to nonlinear dynamical systems in astrodynamics problems and to reachable sets. The attractive or reachable set using optimal control is characterized by the solution to the Hamilton-Jacobi-Bellman partial differential equation. A power series approach is used to obtain an approximate solution to the Hamilton-Jacobi-Bellman partial differential equation. The higher order terms are successively obtained starting from the solution to the linearized equation along an equilibrium point or a periodic orbit. We demonstrate our approach through optimal transfer problems in Hill's three-body problem to equilibrium points and to three-dimensional Halo orbits. By using target sets associated with hyperbolic solutions in the Hill problem, we enable the extension of classical invariant manifold techniques to optimal low-thrust transfers. For the non-linear sets, we are also able to compute transfers between specific periodic or equilibrium solutions, and thus utilize both the stable and unstable manifolds in these systems.

\section{References}

[1] Howell, K., Barden, B. T., and Lo, M. W., "Application of Dynamical Systems Theory to Trajectory Design for a Libration Point Mission," Journal of the Astronautical Sciences, Vol. 45, No. 2, 1997, pp. 161-178.

[2] Gómez, G., Llibre, J., Martınez, R., and Simó, C., Dynamics and Mission Design Near Libration Point Orbits-Volume 1: Fundamentals: The Case of Collinear Libration Points. World Scientific, London, 2001.

[3] Gómez, G., Koon, W., Lo, M. W., Marsden, J. E., Masdemont, J., and Ross, S. D., "Invariant manifolds, the Spatial Three-Body Problem and Space Mission Design," Advances in the Astronautical Sciences, Vol. 109, pp. 3-22.

[4] Koon, W. S., Lo, M. W., Marsden, J. E., and Ross, S. D., "The Genesis Trajectory and Heteroclinic Cycles," Proceedings of the AAS/AIAA Astrodynamics Specialist Conference, 1999.

[5] Koon, W. S., Lo, M. W., Marsden, J. E., and Ross, S. D., "Heteroclinic connections between periodic orbits and resonance transitions in celestial mechanics," Chaos: An Interdisciplinary Journal of Nonlinear Science, Vol. 10, No. 2, 2000, pp. 427-469.

[6] Koon, W. S., Lo, M. W., Marsden, J. E., and Ross, S. D., "Low energy transfer to the Moon," Dynamics of Natural and Artificial Celestial Bodies, pp. 63-73, Springer, 2001. 
[7] Parker, J. S. and Anderson, R. L., Low-Energy Lunar Trajectory Design. John Wiley \& Sons, 2014.

[8] Davis, K. E., Anderson, R. L., Scheeres, D. J., and Born, G. H., "The Use of Invariant Manifolds for Transfers Between Unstable Periodic Orbits of Different Energies," Celestial Mechanics and Dynamical Astronomy, Vol. 107, No. 4, 2010, pp. 471-485.

[9] Davis, K. E., Anderson, R. L., Scheeres, D. J., and Born, G. H., “Optimal Transfers Between Unstable Periodic Orbits Using Invariant Manifolds,” Celestial Mechanics and Dynamical Astronomy, Vol. 109, No. 3, 2011, pp. 241-264.

[10] Bando, M. and Scheeres, D. J., "Attractive Sets to Unstable Orbits Using Optimal Feedback Control," Journal of Guidance, Control, and Dynamics, 2016, pp. 2725-2739.

[11] Rayman, M. D., Varghese, P., Lehman, D. H., and Livesay, L. L., "Results from the Deep Space 1 Technology Validation Mission,” Acta Astronautica, Vol. 47, No. 2, 2000, pp. 475-487.

[12] Kawaguchi, J., Fujiwara, A., and Uesugi, T., "Hayabusa! =ts technology and science accomplishment summary and Hayabusa-2," Acta Astronautica, Vol. 62, No. 10, 2008, pp. 639-647.

[13] Russell, C. and Raymond, C., “The Dawn Mission to Vesta and Ceres," Space Science Reviews, Vol. 163, No. 1-4, 2011, pp. 3-23.

[14] Saridis, G. N. and Lee, C.-S. G., “An Approximation Theory of Optimal Control for Trainable Manipulators," IEEE Transactions on Systems, Man and Cybernetics, Vol. 9, No. 3, 1979, pp. 152-159.

[15] Beard, R. W., Saridis, G. N., and Wen, J. T., "Galerkin Approximations of the Generalized HamiltonJacobi-Bellman Equation,” Automatica, Vol. 33, 1997, pp. 2159-2178.

[16] Lukes, D. L., “Optimal Regulation of Nonlinear Dynamical Systems," SIAM Journal on Control, Vol. 7, No. 1, 1969, pp. 75-100.

[17] Guibout, V. M. and Scheeres, D. J., "Solving Relative Two-Point Boundary Value Problems: Spacecraft Formulation Flight Transfers Application,” Journal of Guidance, Control, and Dynamics, Vol. 27, No. 4, 2004, pp. 693-704.

[18] Park, C., Guibout, V. M., and Scheeres, D. J., "Solving Optimal Continuous Thrust Rendezvous Problems with Generating Functions," Journal of Guidance, Control, and Dynamics, Vol. 29, No. 2, 2006, pp. 321-331. 
[19] Vadali, S. R. and Sharma, R., "Optimal finite-time feedback controllers for nonlinear systems with terminal constraints," Journal of Guidance, Control, and Dynamics, Vol. 29, No. 4, 2006, pp. 921928.

[20] Willemstein, A. P., “Optimal Regulation of Nonlinear Dynamical Systems on a Finite Interval," SIAM Journal on Control and Optimization, Vol. 15, No. 6, 1977, pp. 1050-1069.

[21] Holzinger, M. J. and Scheeres, D. J., "Reachability Results for Nonlinear Systems with Ellipsoidal Initial Sets," IEEE Transactions on Aerospace and Electronic Systems, Vol. 48, No. 2, 2012, pp. 15831600.

[22] Mitchell, I. M., B., A. M., and Tomlin, C. J., “A Time-Dependent Hamilton-Jacobi Formulation of Reachable Sets for Continuous Dynamic Games," IEEE Transactions on Automatic Control, Vol. 50, No. 7, 2005, pp. 947-957.

[23] Scheeres, D. J., Orbital Motion in Strongly Perturbed Environments. 2012, pp. 306-307, 312-316.

[24] Szebehely, V., Theories of Orbits: The Restricted Problem of Three Bodies. 1967, pp.602-629. 